\title{
PREFACE TO SPECIAL ISSUE MULTIPHASE FLOWS AND HEAT/MASS TRANSFER
}

This issue is devoted to the problems of Multiphase Flows and Heat/Mass Transfer. Three papers included in this issue concern the theoretical investigations on the behavior of nonuniform hydrodynamical systems under the action of time-dependent external fields. The effects of pulsating pressure field, external mechanical vibrations, and alternating electric field are discussed.

In the paper by A.A. Alabuzhev, the dynamics of a cylindrical gaseous bubble surrounded by a coaxial layer of incompressible fluid of different density and bounded in the axial direction by parallel rigid plates with different properties are studied. The system is subjected to the pulsating pressure field. Velocity of three-phase contact line motion is assumed to be proportional to the contact angle deviations. It is shown that, in some parameter ranges, the frequency of volume oscillations can be close to zero. If this frequency is close to one of the frequencies of shape oscillations, then a double resonance response is observed that leads the unlimited growth of oscillation amplitude independently of the coefficient of eigen-oscillation damping.

E.S. Sadilov discusses the effect of normal vibrations on the stability of a three-layer system of fluids in zero gravity conditions. It is found that, when the ratio of external layer thicknesses tends to unity the critical vibration amplitude for subharmonic instability mode tends to infinity, which means the disappearance of subharmonic instability mode for the external layers of equal thicknesses. Synchronous instability mode exists at any ratio of the external layer thicknesses.

The work "Electroconvection Instability of Poorly Conducting Fluid in Alternating Electric Field," by N.N. Kartavykh and O.O. Nekrasov, deals with the study of a flat horizontal layer of a poorly conducting fluid subjected to the alternating electric field and heating from above. The electroconductive instability mechanism is analyzed. On the basis of the linear analysis, a map of the stability of a conductive state of nonuniformly poorly heated conducting fluid is plotted and the dependence of the critical wave numbers on the electric field frequency is obtained. Quasiperiodic, synchronous, and chaotic oscillation regimes are found as a result of the nonlinear system dynamics analysis. The domains of coexistence of oscillatory modes with different intensities of flows are found.

In the work "Investigation of the Isothermal Rheokinetic Polymerization of the Epoxy Oligomer," by V.G. Gilev, V.S. Chudinov, S.V. Rusakov, and A.V. Kondyurin, an experimental study and numerical simulations of the kinetics of polymerization of the epoxy oligomer were carried out. Numerical analysis of the kinetic dependencies of viscosity allows one to estimate the reaction rate constants of the polymerization process, which makes it possible to predict the properties of the polymer during its formation. It is shown that the results of viscosity measurements can track quite well, not only the curing process of the binder but also its rate.

The article by K. Schweikert, A. Sielaff, and P. Stephan, "Heat Flux during Dip-Coating of a Superheated Substrate," is devoted to the transient heat flux calculations based on temperature measurements during the process of dipcoating of a superheated substrate. The surface temperature of the solid substrate is measured using high-resolution infrared thermography. Then, the obtained values were used as a boundary condition in simulations at the interface between the superheated substrate and the fluid. Two evaporation regimes, namely, contact line evaporation and microlayer evaporation, were clearly distinguished by their magnitude in overall heat flux.

The outflow of superheated fluid from the pipe channel into a closed volume is numerically simulated in the paper by M. Alekseev, I. Vozhakov, and S. Lezhnin. The simulations were carried out on the basis of a homogeneous onevelocity model. Distributions of pressure, velocity, and steam content in the volume are obtained. It was revealed that the distance between the pipe channel and the opposite wall affects the time to reach quasistationary values for the pressure and steam content.

In the work by T. Gambaryan-Roisman, "Simultaneous Imbibition and Evaporation of Liquids on Grooved Substrates," the hydrodynamics and heat and mass transport processes on textured surfaces are described theoretically and 
numerically. A typical element of the model system is a single groove, along which the liquid flows under the action of capillary pressure gradient.

The paper "Heat Transfer Enhancement in Superheated Hydrocarbons with Traces of Water: The Effect of Pressure," by K.V. Lukianov, A.N. Kotov, A.A. Starostin, and P.V. Skripov, is devoted to experimental investigations of this process. The effect of enhancement of the heat transfer through the probe surface has been revealed for specific pulse heating of a probe.

\section{Guest Editors:}

Tatyana Lyubimova

Institute of Continuous Media Mechanics,

Ural Branch of Russian Academy of Science,

Perm, 614013, Russia

Alexander Kupershtokh

Lavrentyev Institute of Hydrodynamics

Siberian Branch of Russian Academy of Science,

Novosibirsk, 630090, Russia
Olga Goncharova

Altai State University,

Barnaul, 656049, Russia 